\title{
PRIORIZACIÓN DE LAS FUNCIONES DEL SISTEMA DE INNOVACIÓN TECNOLÓGICA DE LA TRANSFORMACIÓN DIGITAL DEL SECTOR OLIVARERO DE ANDALUCÍA: VISIÓN DE LOS AGENTES DE GENERACIÓN DE CONOCIMIENTO
}

\author{
$\underline{\text { Liliana Reina }}^{a}$, Carlos Parra $^{b^{*}}$, Carmen Carmona $^{b}$, Samir Sayadi $^{b}$ \\ ${ }^{a}$ Universidad de Córdoba; Dpto. Economía, Sociología y Política Agraria (Córdoba, z52reusm@uco.es). \\ ${ }^{b}$ Instituto de Investigación y Formación Agraria y Pesquera (IFAPA); Área de Economía de la Cadena \\ Alimentaria; Centro Camino de Purchil (Granada, carlos.parra@juntadeandalucia.es, \\ mariac.carmona.torres@juntadeandalucia.es, samir.sayadi@juntadeandalucia.es).
}

\begin{abstract}
Resumen
Este trabajo analiza la importancia que atribuyen los agentes de generación de conocimiento (universidades, centros de investigación y empresas de soluciones digitales) a un conjunto de siete funciones y 33 subfunciones que se consideran necesarias para un buen funcionamiento del Sistema de Innovación Tecnológica (SIT) asociado a la Transformación Digital (TD) del sector olivarero andaluz. Para ello, se define y evalúa un modelo multicriterio mediante la metodología del Proceso Analítico Jerárquico (AHP). Los resultados indican que entre las funciones más importantes para evaluar el funcionamiento del SIT se encuentran las actividades empresariales y la transferencia de conocimiento; y a nivel de subfunciones la disponibilidad de las opciones tecnológicas, la suficiencia del tamaño de mercado potencial y la adaptación a la legislación vigente. La función de orientación de la investigación es la que menor prioridad tiene para evaluar el desempeño del SIT; y a nivel de subfunciones las relacionadas con los espacios de participación y coordinación entre los agentes y las normas y reglamentos.

Palabras clave: Sistema de innovación tecnológica, digitalización, agentes de innovación, AHP, olivar.
\end{abstract}

\section{Introducción}

Los Sistemas de Innovación Tecnológica (SIT) se definen como el conjunto de redes de actores e instituciones que interactúan conjuntamente en un campo tecnológico específico y que contribuyen a la generación, difusión y utilización de una nueva tecnología (Markard and Truffer, 2008). La esencia del enfoque SIT, como aproximación sistémica al estudio de la innovación, consiste en combinar un análisis de los componentes estructurales que constituyen el sistema con un análisis de las principales funciones del mismo (Bergek et al., 2008). Las funciones de un SIT son los tipos de actividades que deben darse dentro del sistema para su adecuado funcionamiento (Bergek et al., 2015, 2008; Markard et al., 2015). Acorde con Bergek et at. (2008) existen siete funciones clave: 1) actividades empresariales, 2) desarrollo de conocimientos, 3) transferencia de conocimientos, 4) orientación de la investigación, 5) formación de mercados, 6) movilización de recursos y 7) creación de legitimidad. El grado de desempeño de las siete funciones propuestas en un SIT está influido por la presencia y la excelencia de los elementos estructurales. Todos los aspectos de la estructura económica y la estructura institucional que afectan al aprendizaje, la búsqueda y la exploración de innovaciones, se definen como elementos estructurales del SIT (Wieczorek and Hekkert, 2012). La presente comunicación analiza la importancia que atribuyen diferentes agentes de generación de conocimiento (universidades, centros de investigación y empresas digitales) a las funciones (y subfunciones) del SIT asociado a la TD del sector olivarero en Andalucía.

\section{Metodología}

Se ha utilizado la metodología de toma de decisiones multicriterio Proceso Analítico Jerárquico (AHP, por sus siglas en inglés), que propone la descomposición de un problema de toma de decisiones por medio de la definición de una estructura jerárquica. Esto permite subdividir un objetivo complejo en un conjunto de subobjetivos más sencillos y determinar cómo influyen cada uno de esos atributos individuales en el objetivo de la decisión (Saaty, 2002, 1990). El modelo AHP en esta investigación consta de las diferentes funciones y subfunciones de un SIT. Estas se han definido a partir de la revisión de la literatura científica (Bach et al., 2020; Bergek et al., 2008; Edsand, 2019; Liu et al., 2018; Markard et al., 2015; Planko et al., 2017; Stephan et al., 2017; van der Loos et al., 2020). El modelo final consta de 3 niveles (Figura 1):

- Nivel I: Corresponde al objetivo principal, o meta, que se pretende alcanzar al resolver el problema de toma de decisiones. En este caso, evaluar el desempeño funcional del SIT de la TD del sector olivarero andaluz. 
- Nivel II: Consta de las siete funciones clave de un SIT: Actividades empresariales, desarrollo de conocimiento, transferencia de conocimiento, orientación de la investigación, formación de mercado, movilización de recursos y creación de legitimidad.

- Nivel III: Se corresponde con las subfunciones, que son los ítems específicos dentro de cada función, como se detalla en la Tabla 1.

Tabla 1. Modelo AHP para la evaluación del desempeño funcional del SIT de la TD en el sector

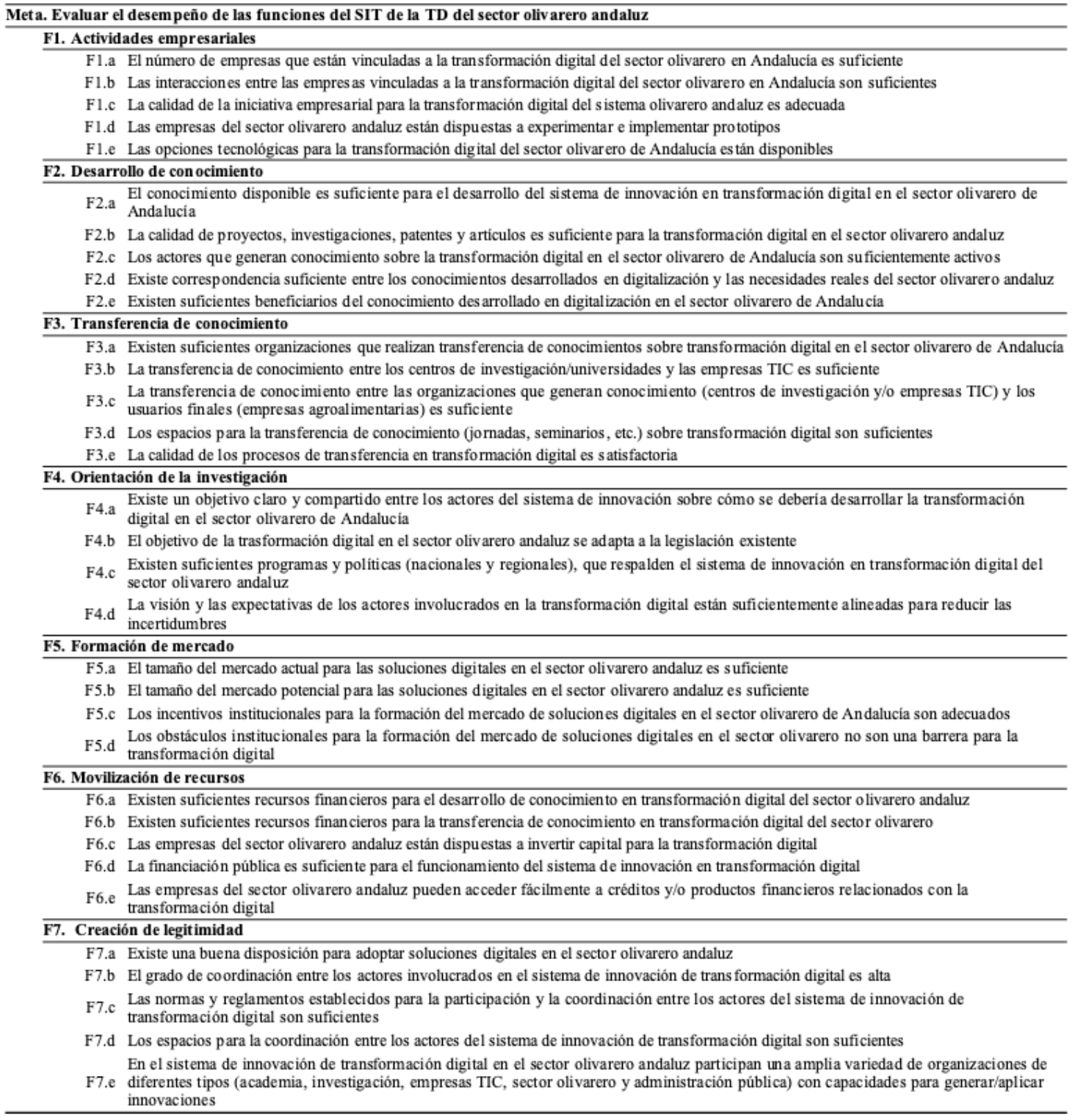

Una vez definido el modelo se evalúa la prioridad (o peso) de cada una de las funciones y subfunciones con respeto a los elementos de los que dependen. AHP permite realizar una evaluación cuantitativa de los elementos de decisión, en nuestro caso funciones y subfunciones, permitiendo priorizarlos en una escala de razón y haciéndolos conmensurables y comparables (Forman and Selly, 2001; Kurttila et al., 2000; ParraLópez et al., 2009). Para evaluar el modelo se utilizó el conocimiento experto debido a la baja disponibilidad de datos objetivos para Andalucía y la naturaleza compleja de los temas investigados. En particular, se entrevistó a 19 expertos con diversos perfiles y experiencia en el tema ( 14 de organizaciones de I+D y 5 de empresas de soluciones digitales) de agosto a octubre de 2020, siguiendo un cuestionario estructurado. Se ha utilizado una puntuación directa (direct rating) (Calabrese et al., 2019; Forman and Selly, 2001), en una escala de 1 (muy poca prioridad/desempeño) a 9 (alta prioridad/desempeño) (Carmona-Torres et al., 2014) 


\section{Resultados}

\subsection{Priorización global de las funciones del SIT}

Los resultados presentados aquí se refieren a las prioridades globales, obtenidas como media de los grupos de interés para cada función y subfunción, ponderando por igual las preferencias de todos los grupos. La Figura 1 muestra que, en el SIT de la TD, los agentes generadores de conocimiento le atribuyen la mayor importancia para el buen funcionamiento del sistema a la Función 1. Actividades empresariales, seguida de la Función 3. Transferencia de conocimiento. Entre las menos priorizadas se encuentran la Función 4. Orientación de la investigación, y la Función 5. Formación del mercado.

Gráfico 1. Priorización global de las funciones

\begin{tabular}{ll}
\multicolumn{1}{c}{ Funciones } & Priorización \\
\hline F1 Actividades empresariales & \\
F2 Desarrollo de conocimiento & 0,1545 \\
F3 Transferencia de conocimiento & 0,1469 \\
F4 Orientación de la investigación & 0,1535 \\
F5 Formación de mercado & 0,1312 \\
F6 Movilización de recursos & 0,1333 \\
F7 Creación de legitimidad & 0,1417 \\
\hline
\end{tabular}

Gráfico 2. Priorización global de las subfunciones

\begin{tabular}{|c|c|c|}
\hline \multirow{5}{*}{$\begin{array}{c}\text { F1. } \\
\text { Actividades } \\
\text { empresariales }\end{array}$} & F1.a & 0,0293 \\
\hline & F1.b & 0,0260 \\
\hline & F1.c & 0,0313 \\
\hline & F1.d & 0,0287 \\
\hline & F1.e & 0,0393 \\
\hline \multirow{5}{*}{$\begin{array}{c}\text { F2. } \\
\text { Desarrollo de } \\
\text { conocimiento }\end{array}$} & F2.a & 0,0311 \\
\hline & F2.b & 0,0325 \\
\hline & F2.c & 0,0265 \\
\hline & F2.d & 0,0288 \\
\hline & F2.e & 0,0279 \\
\hline \multirow{5}{*}{$\begin{array}{c}\text { F3. } \\
\text { Transferencia de } \\
\text { conocimiento }\end{array}$} & F3.a & 0,0306 \\
\hline & F3.b & 0,0302 \\
\hline & F3.c & 0,0326 \\
\hline & F3.d & 0,0278 \\
\hline & F3.e & 0,0321 \\
\hline \multirow{4}{*}{$\begin{array}{c}\text { F4. } \\
\text { Orientación de la } \\
\text { investigación }\end{array}$} & F4.a & 0,0318 \\
\hline & F4.b & 0,0366 \\
\hline & F4.c & 0,0315 \\
\hline & F4.d & 0,0313 \\
\hline \multirow{4}{*}{$\begin{array}{c}\text { F5. } \\
\text { Formación de mercado }\end{array}$} & F5.a & 0,0322 \\
\hline & F5.b & 0,0367 \\
\hline & F5.c & 0,0301 \\
\hline & F5.d & 0,0343 \\
\hline \multirow{5}{*}{$\begin{array}{l}\text { F6. } \\
\text { Movilización de } \\
\text { recursos }\end{array}$} & F6.a & 0,0296 \\
\hline & F6.b & 0,0267 \\
\hline & F6.c & 0,0289 \\
\hline & F6.d & 0,0266 \\
\hline & F6.e & 0,0300 \\
\hline \multirow{5}{*}{$\begin{array}{c}\text { F7. } \\
\text { Creación de } \\
\text { legitimidad }\end{array}$} & F7.a & 0,0301 \\
\hline & F7.b & 0,0294 \\
\hline & F7.c & 0,0246 \\
\hline & F7.d & 0,0242 \\
\hline & F7.e & 0,0307 \\
\hline
\end{tabular}

\subsection{Priorización global de las subfunciones del SIT}

El orden de las prioridades o pesos de las subfunciones (Figura 2) sitúan en primer lugar a F1.e Las opciones tecnológicas para la transformación digital del sector olivarero de Andalucía están disponibles. Le siguen en orden de importancia F5.b El tamaño del mercado potencial para las soluciones digitales en el sector olivarero andaluz es suficiente; F4.b. El objetivo de la trasformación digital en el sector olivarero andaluz se adapta a la legislación existente; y F5.d Los obstáculos institucionales para la formación del mercado de soluciones digitales en el sector olivarero no son una barrera para la transformación digital. Las subfunciones que menor prioridad han obtenido son: F7.d Los espacios para la coordinación entre los actores del sistema de innovación de la transformación digital son suficientes; F7.c Las normas y reglamentos establecidos para la participación y la coordinación entre los actores del sistema de innovación de la transformación digital son suficientes; F1.b Las interacciones entre las empresas vinculadas a la TD del sector olivarero andaluz son suficientes; y F2.c Los actores que generan conocimiento sobre la transformación digital en el sector olivarero de Andalucía son suficientemente activos.

\section{Conclusiones}

La evaluación del Sistema de Innovación Tecnológica (SIT) de la Transformación Digital del sector olivarero en Andalucía indica que la función con la mayor importancia o prioridad para evaluar el adecuado funcionamiento del SIT es la de actividades empresariales, y la subfunción más importante es la que se refiere a la disponibilidad de las opciones tecnológicas para la TD del sector olivarero de Andalucía. Por otra parte, la que menor importancia tiene es la función de orientación a la investigación, y las subfunciones relacionadas con la coordinación de los actores y la suficiencia de normas para la participación en el SIT. 
Finalmente, indicar que este trabajo abre un abanico de líneas para futuras investigaciones en lo referido al desarrollo completo de la red de decisión, incluyendo la evaluación del funcionamiento del SIT en cada una de las subfunciones y funciones y a nivel global. También puede ser interesante la aplicación del modelo diseñado a otros grupos de interés del sector en Andalucía, así como en otros sectores del sistema alimentario andaluz, y en otros territorios con cultivo de olivar, con el objetivo de identificar si existen variaciones en la valoración del funcionamiento de los SIT que pudieran estar relacionadas con la variable territorial o sectorial.

\section{Bibliografía}

Bach, H., Bergek, A., Bjørgum, Ø., Hansen, T., Kenzhegaliyeva, A., Steen, M., (2020). "Implementing maritime battery-electric and hydrogen solutions: A technological innovation systems analysis". Transp. Res. Part D Transp. Environ. 87, 102492.

Bergek, A., Hekkert, M., Jacobsson, S., Markard, J., Sandén, B., Truffer, B., (2015). “Technological innovation systems in contexts: Conceptualizing contextual structures and interaction dynamics". Environ. Innov. Soc. Transit. 16, 51-64.

Bergek, A., Jacobsson, S., Carlsson, B., Lindmark, S., Rickne, A., (2008). “Analyzing the functional dynamics of technological innovation systems: A scheme of analysis”. Res. Policy 37, 407-429.

Calabrese, A., Costa, R., Levialdi, N., Menichini, T., (2019). "Integrating sustainability into strategic decisionmaking: A fuzzy AHP method for the selection of relevant sustainability issues". Technol. Forecast. Soc. Change.

Carmona-Torres C., Parra-López C., Hinojosa-Rodríguez A., and Sayadi S. (2014). "Farm-level multifunctionality associated with farming techniques in olive growing: An integrated modeling approach". Agricultural Systems, 127: p. 97-114.

Edsand, H.E., (2019). "Technological innovation system and the wider context: A framework for developing countries”. Technol. Soc. 58, 101150. https://doi.org/10.1016/j.techsoc.2019.101150

Forman, E.H., Selly, M.A., (2001). Decision by Objective: How to Convince Others That You are Right.

Kurttila, M., Pesonen, M., Kangas, J., Kajanus, M., (2000). "Utilizing the analytic hierarchy process (AHP) in SWOT analysis - A hybrid method and its application to a forest-certification case". For. Policy Econ. 1, $41-52$.

Liu, G., Gao, P., Chen, F., Yu, J., Zhang, Y., (2018). "Technological innovation systems and IT industry sustainability in China: A case study of mobile system innovation". Telemat. Informatics 35, 1144-1165.

Markard, J., Hekkert, M., Jacobsson, S., (2015). "The technological innovation systems framework: Response to six criticisms". Environ. Innov. Soc. Transitions 16, 76-86. https://doi.org/10.1016/j.eist.2015.07.006

Markard, J., Truffer, B., (2008). "Technological innovation systems and the multi-level perspective: Towards an integrated framework". Res. Policy 37, 596-615. https://doi.org/10.1016/j.respol.2008.01.004

Parra-López, C., Calatrava-Requena, J., (2006). "Comparison of farming techniques actually implemented and their rationality in organic and conventional olive groves in Andalusia, Spain”. Biol. Agric. Hortic. 24, 3559.

Planko, J., Cramer, J., Hekkert, M.P., Chappin, M.M.H., (2017). "Combining the technological innovation systems framework with the entrepreneurs' perspective on innovation”. Technol. Anal. Strateg. Manag. 29, 614-625. https://doi.org/10.1080/09537325.2016.1220515

Saaty, T.L., (2002). Decision making with the Analytic Hierarchy Process. Sci. Iran. https://doi.org/10.1504/ijssci.2008.017590

Saaty, T.L., (1990). "How to make a decision: The analytic hierarchy process". Eur. J. Oper. Res.

Stephan, A., Schmidt, T.S., Bening, C.R., Hoffmann, V.H., (2017). "The sectoral configuration of technological innovation systems: Patterns of knowledge development and diffusion in the lithium-ion battery technology in Japan". Res. Policy 46, 709-723. https://doi.org/10.1016/j.respol.2017.01.009

van der Loos, A., Normann, H.E., Hanson, J., Hekkert, M.P., (2020). "The co-evolution of innovation systems and context: Offshore wind in Norway and the Netherlands". Renew. Sustain. Energy Rev. 138, 110513.

Wieczorek, A.J., Hekkert, M.P., (2012). "Systemic instruments for systemic innovation problems: A framework for policy makers and innovation scholars". Sci. Public Policy 39, 74-87.

https://doi.org/10.1093/scipol/scr008

\section{Agradecimientos}

Los autores desean expresar su agradecimiento por el apoyo financiero recibido del Instituto de Investigación y Formación Agraria y Pesquera (IFAPA), a través del proyecto de investigación "Transformación digital del sector olivarero en Andalucía: Análisis sistémico, estructural y funcional para favorecer su desarrollo (digitalOli)" (PR.AVA.AVA2019.009), cofinanciado en un 80\% por el Fondo Europeo de Desarrollo Regional dentro del Programa Operativo FEDER de Andalucía 2014-2020. 